\title{
Mycobacterium abscessus Subspecies massiliense
}

National Cancer Institute

\section{Source}

National Cancer Institute. Mycobacterium abscessus Subspecies massiliense. NCI

Thesaurus. Code C150882.

A subspecies of Mycobacterium abscessus. M. abcessus subsp. massiliense has a truncated erm(41) gene and is susceptible to clarithromycin. 